\title{
SOME POSSIBLY DEGENERATE ELLIPTIC PROBLEMS WITH MEASURE DATA AND NON LINEARITY ON THE BOUNDARY
}

\author{
THIERRY GALLOUËT AND YANNICK SIRE
}

\begin{abstract}
The goal of this paper is to study some possibly degenerate elliptic equation in a bounded domain with a nonlinear boundary condition involving measure data. We investigate two types of problems: the first one deals with the laplacian in a bounded domain with measure supported on the domain and on the boundary. A second one deals with the same type of data but involves a degenerate weight in the equation. In both cases, the nonlinearity under consideration lies on the boundary. For the first problem, we prove an optimal regularity result, whereas for the second one the optimality is not guaranteed but we provide however regularity estimates.
\end{abstract}

RÉSumÉ. Le but de cet article est l'étude d'équations elliptiques pouvant dégénérer, à données mesures, dans un domaine borné, et avec nonlinéarité au bord du domaine. On étudie deux types de problèmes : un permier est une équation elliptique non dégnérée dans un domaine borné avec des doinnées mesures, supportées à la fois à l'intérieur du domaine et sur le bord de celui-ci. On traite dans une deuxième partie un problème elliptique dégénéré. On établit des résultat d'existence et de régularité dans les deux cas. Dans les deux problèmes considérés, la nonlinéarité est au bord du domaine.

\section{Contents}

1. Introduction

2. The case $\alpha=0$ and problem (1.1)

2.1. Regularization of the boundary problem

2.2. Estimates on the regularized problem

2.3. Passage to the limit in the regularized problem

$T G$ : Université Aix-Marseille $1-$ LATP - Marseille, France gallouet@cmi.univ-mrs.fr

YS: Université Aix-Marseille 3, Paul Cézanne - LATP - Marseille, France sire@cmi. univ-mrs.fr. 
3. The degenerate case $\alpha \neq 0$ and problem (1.2) 10

3.1. Regularization $\quad 10$

3.2. Estimates 10

4. Extensions of the previous result $\quad 12$

References 13

\section{INTRODUCTION}

Let $\Omega$ be a smooth bounded open subset of $\mathbb{R}^{N}$ for $N \geq 2$. Let $\left\{\Gamma_{1}, \Gamma_{2}\right\}$ be a measurable partition of $\partial \Omega$ such that $\left|\Gamma_{1}\right|>0$. Consider $\mu_{1} \in \mathcal{M}(\bar{\Omega})$ and $\mu_{2} \in \mathcal{M}(\partial \Omega)$, two Radon measures supported on $\Omega$ and $\Gamma_{2}$ respectively.

The paper is devoted to the study of the two following problems for $\gamma>1$

$$
\begin{gathered}
\left\{\begin{array}{c}
\Delta u=\mu_{1} \text { in } \Omega, \\
u=0 \text { on } \Gamma_{1}, \\
\partial_{\nu} u=\mu_{2}-|u|^{\gamma-1} u \text { on } \Gamma_{2} .
\end{array}\right. \\
\left\{\begin{array}{c}
\nabla \cdot\left(d(x, \partial \Omega)^{\alpha} \nabla\right) u=\mu_{1} \text { in } \Omega, \\
u=0 \text { on } \Gamma_{1}, \\
d(x, \partial \Omega)^{\alpha} \partial_{\nu} u=\mu_{2}-|u|^{\gamma-1} u \text { on } \Gamma_{2} .
\end{array}\right.
\end{gathered}
$$

where $\alpha \in(-1,1)$ and $d(x, \partial \Omega)$ denotes the distance from a point $x \in \Omega$ to the boundary $\partial \Omega$. Notice that when $\alpha=0$, problem (1.2) reduces to (1.1). The weight $d(x, \partial \Omega)^{\alpha}$ degenerates at the boundary either by explosion for $\alpha<0$ or to 0 for $\alpha>0$. We will see that there is a difference, as far as regularity is concerned, between the cases $\alpha \neq 0$ and $\alpha=0$ and we will deal with these problems seperately.

Several works have been devoted to the study of elliptic equations with non smooth data. Particularly, the following equation

$$
-\Delta u+|u|^{\gamma-1} u=f \in L^{1} \text { in } \Omega
$$

with Dirichlet boundary conditions has been investigated by several authors starting with the works of Brézis and Strauss [4]. In the case of $\Omega=\mathbb{R}^{N}$, we refer the reader to the works [1] and [8]. In this latter work, the authors investigate the range $\gamma \leq 1$, and prove that a growth condition on $f$ is necessary to ensure existence and uniqueness. The case of the $p$-laplacian instead of the laplacian has been investigated in [3].

When $f$ is a measure, the picture is more complicated. In [2], the authors solved the existence and regularity problem for measure data in 
smooth domains for Leray-Lions operator of the type $-\nabla \cdot a(x, u, \nabla u)$. However one can prove existence and regularity if and only if $f \in$ $L^{1}(\Omega)+W^{-2, \gamma}(\Omega)$. This latter condition is equivalent to $|f|(A)=0$ for every borelian susbet $A$ of $\Omega$ having zero $W^{2, \gamma^{\prime}}$-capacity (here $\gamma^{\prime}$ is the conjugate exponent of $\gamma$ ).

Under some assumptions on $\gamma$, we prove that our problems (1.1)-(1.2) admit a solution and we study their regularity.

The motivation to investigate the degenerate problem (1.2) comes from recent investigations on non local operators. Indeed, the following result has been proved by Caffarelli and Silvestre (see [5]): Given $s \in$ $(0,1)$, let $\alpha=1-2 s \in(-1,1)$. Using variables $(x, y) \in \mathbb{R}_{+}^{n+1}:=$ $(0,+\infty) \times \mathbb{R}^{N}$, the space $H^{s}\left(\mathbb{R}^{N}\right)$ coincides with the trace on $\partial \mathbb{R}_{+}^{N+1}$ of

$$
H^{1}\left(x^{\alpha}\right):=\left\{u \in H_{l o c}^{1}\left(\mathbb{R}_{+}^{N+1}\right): \int_{\mathbb{R}_{+}^{n+1}} x^{\alpha}\left(u^{2}+|\nabla u|^{2}\right) d x d y<+\infty\right\} .
$$

In other words, given any function $u \in H^{1}\left(x^{\alpha}\right) \cap C\left(\overline{\mathbb{R}_{+}^{N+1}}\right), v:=$ $\left.u\right|_{\partial \mathbb{R}_{+}^{N+1}} \in H^{s}\left(\mathbb{R}^{N}\right)$ and there exists a constant $C=C(n, s)>0$ such that

$$
\|v\|_{H^{s}\left(\mathbb{R}^{N}\right)} \leq C\|u\|_{H^{1}\left(x^{\alpha}\right)} .
$$

So, by a standard density argument (see [6]), every $u \in H^{1}\left(x^{\alpha}\right)$ has a well-defined trace $v \in H^{s}\left(\mathbb{R}^{N}\right)$. Conversely, any $v \in H^{s}\left(\mathbb{R}^{N}\right)$ is the trace of a function $u \in H^{1}\left(x^{\alpha}\right)$. In addition, the function $u \in H^{1}\left(x^{\alpha}\right)$ defined by

$$
u:=\arg \min \left\{\int_{\mathbb{R}_{+}^{N+1}} x^{\alpha}|\nabla w|^{2} d x:\left.w\right|_{\partial \mathbb{R}_{+}^{N+1}}=v\right\}
$$

solves the PDE

$$
\left\{\begin{aligned}
\operatorname{div}\left(x^{\alpha} \nabla u\right) & =0 & & \text { in } \mathbb{R}_{+}^{N+1} \\
u & =v & & \text { on } \partial \mathbb{R}_{+}^{N+1} .
\end{aligned}\right.
$$

By standard elliptic regularity, $u$ is smooth in $\mathbb{R}_{+}^{N+1}$. It turns out that $x^{\alpha} u_{x}(x, \cdot)$ converges in $H^{-s}\left(\mathbb{R}^{N}\right)$ to a distribution $f \in H^{-s}\left(\mathbb{R}^{N}\right)$, as $x \rightarrow 0^{+}$i.e. $u$ formally solves

$$
\left\{\begin{aligned}
\operatorname{div}\left(x^{\alpha} \nabla u\right)=0 & \text { in } \mathbb{R}_{+}^{N+1} \\
-x^{\alpha} u_{x}=f & \text { on } \partial \mathbb{R}_{+}^{N+1} .
\end{aligned}\right.
$$

Consider the Dirichlet-to-Neumann operator

$$
\Gamma_{\alpha}:\left\{\begin{aligned}
H^{s}\left(\mathbb{R}^{N}\right) & \rightarrow H^{-s}\left(\mathbb{R}^{N}\right) \\
v & \mapsto \Gamma_{\alpha}(v)=f:=-\left.x^{\alpha} u_{x}\right|_{\partial \mathbb{R}_{+}^{N+1}}
\end{aligned}\right.
$$


where $u$ is the solution of (1.3)-(1.5). Given $f \in H^{-s}\left(\mathbb{R}^{N}\right)$, a function $v \in H^{s}\left(\mathbb{R}^{N}\right)$ solves the equation

$$
\frac{1}{d_{N, s}}(-\Delta)^{s} v=f \quad \text { in } \mathbb{R}^{N}
$$

if and only if its lifting $u \in H^{1}\left(x^{\alpha}\right)$ solves $u=v$ on $\partial \mathbb{R}_{+}^{N+1}$ and

$$
\left\{\begin{aligned}
\operatorname{div}\left(x^{\alpha} \nabla u\right)=0 & \text { in } \mathbb{R}_{+}^{N+1} \\
-x^{\alpha} u_{x}=f & \text { on } \partial \mathbb{R}_{+}^{N+1} .
\end{aligned}\right.
$$

Here $d_{N, s}$ is a normalizing constant. Equation (1.6) involves the fractional laplacian, which symbol is a Fourier multiplier $|\xi|^{2 s}$. We refer the reader to [9] for a potential-theoretic study of the fractional laplacian and Riesz kernels.

A quick look at the previous development shows that the weight $x^{\alpha}$ represents the distance of a point $(x, y) \in \mathbb{R}_{+}^{N+1}$ to the boundary $\partial \mathbb{R}_{+}^{N+1}$ to the power $\alpha$. It is then natural to consider a somehow "localized" version of it, namely problem (1.2), as a starting point of regularity study of fractional order operators. We postpone to future work the study of the equation

$$
(-\Delta)^{s}+|u|^{\gamma-1} u=f \text { in } \mathbb{R}^{N}
$$

where $f \in L_{l o c}^{1}\left(\mathbb{R}^{N}\right)$. This problem is more challenging since it requires local estimates, as done for instance in [3] in the case $s=1$. More precisely, we would need local estimates independent of the radius $R$ of the following boundary problem

$$
\left\{\begin{array}{c}
\nabla \cdot\left(x^{\alpha} \nabla u\right)=\mu_{1}, \text { in } B_{R}^{+}, \\
u=0, \text { on } \partial^{+} B_{R}^{+}, \\
x^{\alpha} \partial_{x} u=\mu_{2}-|u|^{\gamma-1} u, \text { on } \partial^{0} B_{R}^{+}
\end{array}\right.
$$

where

$$
\begin{gathered}
B_{R}^{+}=\left\{(x, y) \in \mathbb{R}^{+} \times \mathbb{R}^{N}, \quad|(x, y)|<R\right\}, \\
\partial^{+} B_{R}^{+}=\left\{(x, y) \in \mathbb{R}^{+} \times \mathbb{R}^{N}, \quad|(x, y)|=R\right\}, \\
\partial^{0} B_{R}^{+}=\left\{(0, y), y \in \mathbb{R}^{N},|(0, y)|<R\right\} .
\end{gathered}
$$

It has to be noticed that the weight $d(x, \partial \Omega)^{\alpha}$, since $\alpha \in(-1,1)$, is degenerate at the boundary of the smooth domain $\Omega$. However, this function falls into a specific class of functions, namely $A_{2}$ weights introduced by Muckenhoupt [10]. Indeed, a function $w(x)$ defined on $\mathbb{R}^{N}$ is said to be $A_{2}$ if the following holds: there exists $C>0$ such that

$$
\sup _{B}\left\{\frac{1}{|B|} \int_{B} w(x) d x\right\}\left\{\frac{1}{|B|} \int_{B} w(x)^{-1} d x\right\} \leq C,
$$


for every ball $B \subset \mathbb{R}^{N}$. In our context the $A_{2}$ condition writes

$$
\left\{\frac{1}{R^{n}} \int_{0}^{R}(R-r)^{\alpha} r^{n-1} d r\right\}\left\{\frac{1}{R^{n}} \int_{0}^{R}(R-r)^{-\alpha} r^{n-1} d r\right\} \leq C,
$$

which is clearly satisfied since $\alpha \in(-1,1)$.

The class $A_{2}$ enjoys several very nice properties that we will describe further in the paper. First, we introduce the following notion of weak solution, valid for both problems.

Definition 1.1. A function $u \in W^{1,1}(\Omega)$ is a weak solution of (1.2) (or (1.1) taking $\alpha=0$ ) if for all $\varphi \in C^{\infty}\left(\mathbb{R}^{N}\right)$ with $\varphi=0$ on $\Gamma_{1}$

$$
\int_{\Omega} d(x, \partial \Omega)^{\alpha} \nabla u \cdot \nabla \varphi+\int_{\Omega} \varphi d \mu_{1}=\int_{\Gamma_{2}} \varphi d \mu_{2}-\int_{\Gamma_{2}}|u|^{\gamma-1} u \varphi .
$$

We now state our results.

Our main result is an existence and regularity result for (1.9).

Theorem 1.2. For every $\mu_{1} \in \mathcal{M}(\bar{\Omega}), \mu_{2} \in \mathcal{M}(\partial \Omega)$ supported on $\Omega$ and $\Gamma_{2}$ respectively, $\gamma>1$ and $\alpha=0$, there exists a weak solution $u$ to (1.1) such that

$$
u \in \bigcap_{1<q<\frac{N}{N-1}} W^{1, q}(\Omega) .
$$

As a consequence, the trace $T u$ of $u$ on $\partial \Omega$ satisfies

$$
T u \in \bigcap_{1 \leq q<\frac{N}{N-1}} W^{1-\frac{1}{q}, q}(\partial \Omega)
$$

The previous theorem is optimal in the sense that one can construct measures such that $u \notin W^{1, N / N-1}(\Omega)$. The second one concerns the degenerate case.

Theorem 1.3. For every $\mu_{1} \in \mathcal{M}(\bar{\Omega}), \mu_{2} \in \mathcal{M}(\partial \Omega)$ supported on $\Omega$ and $\Gamma_{2}$ respectively, $\gamma>1$ and $\alpha \in(-1,1)$, there exists a weak solution $u$ to (1.2) such that

$$
u \in \bigcap_{1 \leq q<\frac{2 N+2 \delta(N-1)}{2 N-1+\delta}} W^{1, q}\left(\Omega, d(x, \partial \Omega)^{\alpha}\right)
$$

for some $\delta>0$ depending on $\Omega$ and $\alpha$. As a consequence, the trace Tu of $u$ on $\partial \Omega$ satisfies

$$
T u \in \bigcap_{1<q<\frac{2 N+2 \delta(N-1)}{2 N-1+\delta} .} W^{1-\frac{1+\alpha}{q}, q}(\partial \Omega)
$$


This second theorem is not optimal, as far as regularity is concerned, because of the degeneracy of the weight close to the boundary.

In order to prove Theorems 1.2 1.3 , we proceed in several steps:

- We first approximate the problem with smooth data.

- We obtain estimates with suitable dependence on the data.

- We pass to the limit.

This technique is reminiscent of the technique developped in [2], for instance. In the degenerate case, even if the techniques are similar, this requires new ingredients from the theory of degenerate elliptic equations with $A_{2}$ weights, which have been developped in [7]. For sake of clarity, we will first deal with the problem $\alpha=0$, for which an optimal result can be obtained. In a second part, we will consider $\alpha \neq 0$.

\section{THE CASE $\alpha=0$ AND PROBLEM (1.1)}

2.1. Regularization of the boundary problem. To do so we start by approximating the problem (1.1) and consider it for smooth sequences $\left\{\mu_{1}^{n}\right\}_{n \geq 0},\left\{\mu_{2}^{n}\right\}_{n \geq 0} \subset L^{\infty}$ converging to $\mu_{1}$ and $\mu_{2}$ in the weak* topology, i.e.

$$
\begin{gathered}
\int_{\Omega} \varphi d \mu_{1}^{n} \rightarrow \int_{\Omega} \varphi d \mu_{1}, \quad \forall \varphi \in C(\bar{\Omega}) \\
\int_{\Gamma_{2}} \varphi d \mu_{2}^{n} \rightarrow \int_{\Gamma_{2}} \varphi d \mu_{2}, \quad \forall \varphi \in C(\partial \Omega)
\end{gathered}
$$

The regularized problem then writes weakly

$$
\int_{\Omega} \nabla u_{n} \cdot \nabla \varphi+\int_{\Omega} \varphi \mu_{1}^{n}=\int_{\Gamma_{2}} \varphi \mu_{2}^{n}-\int_{\Gamma_{2}}\left|u_{n}\right|^{\gamma-1} u_{n} \varphi .
$$

We have the following result, as a consequence of an adaptation of standard variational techniques as in [1].

Theorem 2.1. Let $\gamma>1$ and $\mu_{1}^{n}, \mu_{2}^{n}$ as before. There exists a weak solution $H^{1}(\Omega) \bigcap L^{\infty}(\Omega)$ of $(2.10)$.

2.2. Estimates on the regularized problem. We now estimate the solution $u_{n}$ and its gradient $\nabla u_{n}$ with a convenient dependence on the data $\mu_{1}^{n}, \mu_{2}^{n}$.

Lemma 2.2. Let $\gamma>1$ and $u_{n}$ be a weak solution of (2.10) with $\mu_{1}^{n}, \mu_{2}^{n} \in L^{2}\left(\Gamma_{2}\right)$. Then for every $\theta>1$, there exists $C>0$ depending on $\theta$ and on a bound of the $L^{1}$ norm of $\mu_{1}^{n}, \mu_{2}^{n}$ such that 


$$
\left\|u_{n}\right\|_{L^{\gamma}\left(\Gamma_{2}\right)} \leq C
$$

and

$$
\int_{\Omega} \frac{\left|\nabla u_{n}\right|^{2}}{\left(1+\left|u_{n}\right|\right)^{\theta}} \leq C .
$$

Proof. We proof follows the same lines as the one in 3], taking into account that the nonlinearity is on the boundary. Consider the weak formulation of (2.10), i.e.

$$
\int_{\Omega} \nabla u_{n} \cdot \nabla \varphi+\int_{\Omega} \varphi \mu_{1}^{n}=\int_{\Gamma_{2}} \varphi \mu_{2}^{n}-\int_{\Gamma_{2}}\left|u_{n}\right|^{\gamma-1} u_{n} \varphi .
$$

for any $\varphi$ defined as before. We consider a suitable test function for the weak formulation (2.13), by considering for $\theta>0$

$$
\phi_{\theta}(r)=\left\{\begin{array}{l}
\int_{0}^{r} \frac{d t}{(1+t)^{\theta}} \text { if } r \geq 0, \\
-\phi_{\theta}(-r) \text { if } r<0,
\end{array}\right.
$$

Notice that $\phi_{\theta}$ is bounded in $\mathbb{R}$. We plug the following test function in 2.13$)$

$$
\varphi=\phi_{\theta}\left(u_{n}\right)
$$

which is a suitable test function by Stampacchia theorem. This gives, since $\phi_{\theta}$ is bounded,

$$
\int_{\Omega} \frac{\left|\nabla u_{n}\right|^{2}}{\left(1+\left|u_{n}\right|\right)^{\theta}}+\int_{\Gamma_{2}}\left|u_{n}\right|^{\gamma-1} u_{n} \phi_{\theta}\left(u_{n}\right) \leq C\left(\left\|\mu_{1}^{n}\right\|_{L^{1}\left(\Gamma_{2}\right)}+\left\|\mu_{2}^{n}\right\|_{L^{1}(\Omega)}\right) .
$$

This gives the desired result since $\left|u_{n}\right|^{\gamma} \geq C\left|u_{n}\right|^{\gamma-1} u_{n} \phi_{\theta}\left(u_{n}\right)$ for some $C>0$ depending on $\theta$.

From the previous estimates, we deduce regularity estimates on the gradient of $u_{n}$ in some Lebesgue space. This is the object of the following lemma.

Lemma 2.3. Let $\mu_{1}^{n} \in L^{2}(\Omega)$ and $\mu_{2}^{n} \in L^{2}\left(\Gamma_{2}\right)$. Consider $u_{n}$ a weak solution of (2.13) such that

$$
\int_{\Gamma_{2}}\left|u_{n}\right|^{\gamma} \leq C
$$

for some constant $C>0$ and

$$
\int_{\Omega} \frac{\left|\nabla u_{n}\right|^{2}}{\left(1+\left|u_{n}\right|\right)^{\theta}} \leq D
$$


for all $\theta>1$ and some constant $D>0$ depending on $\theta$. Then, for every $q \in\left[1, \frac{N}{N-1}\right)$, there exists $\tilde{C}$ depending on $C, D$ such that

$$
\left\|u_{n}\right\|_{W^{1, q}(\Omega)} \leq \tilde{C} .
$$

Proof. We write, by Hölder inequality for $q \in[1,2)$

$$
\begin{gathered}
\int_{\Omega}\left|\nabla u_{n}\right|^{q}=\int_{\Omega} \frac{\left|\nabla u_{n}\right|^{q}}{\left(1+\left|u_{n}\right|\right)^{\theta q / 2}}\left(1+\left|u_{n}\right|\right)^{\theta q / 2} \leq \\
\left\{\int_{\Omega} \frac{\left|\nabla u_{n}\right|^{2}}{\left(1+\left|u_{n}\right|\right)^{\theta}}\right\}^{q / 2}\left\{\int_{\Omega}\left(1+\left|u_{n}\right|\right)^{\frac{\theta q}{2-q}}\right\}^{\frac{2-q}{2}} \leq \\
C_{1}\left\{\int_{\Omega}\left(1+\left|u_{n}\right|\right)^{\frac{\theta q}{2-q}}\right\}^{\frac{2-q}{2}} .
\end{gathered}
$$

We have for $q^{*}=\frac{q N}{N-q}$

$$
\int_{\Omega}\left(1+\left|u_{n}\right|\right)^{\frac{\theta q}{2-q}} \leq \varepsilon \int_{\Omega}\left|u_{n}\right|^{q^{*}}+C_{2}(\varepsilon)
$$

for every $\varepsilon>0$ provided that $\theta$ is chosen such that $\frac{\theta q}{2-q}<q^{*}$. We now use the Sobolev embeddings to estimate the last term: since $u_{n}=0$ on $\Gamma_{1} \subset \partial \Omega$ and $q \in[1,2)$, we have the Sobolev embedding

$$
\left\|u_{n}\right\|_{L^{q^{*}}(\Omega)} \leq C\left\|\nabla u_{n}\right\|_{L^{q}(\Omega)} .
$$

Hence, chossing $\varepsilon$ small enough, this yields to

$$
\|u\|_{L^{q^{*}}(\Omega)} \leq C_{3}
$$

for some $C_{3}>0$. By observing that one can take $\theta$ arbitrary close to 1 , one gets that

$$
\left\|\nabla u_{n}\right\|_{L^{q}(\Omega)} \leq \tilde{C}
$$

for every $q \in\left[1, \frac{N}{N-1}\right)$.

The previous theorem admits the following corollary by just using the trace embedding.

Corollary 2.4. Let $\mu_{1}^{n}, \mu_{2}^{n}$ as before. Consider $u_{n}$ a weak solution of (2.13) such that

$$
\int_{\Gamma_{2}}\left|u_{n}\right|^{\gamma} \leq C
$$

for some constant $C>0$ and

$$
\int_{\Omega} \frac{\left|\nabla u_{n}\right|^{2}}{\left(1+\left|u_{n}\right|\right)^{\theta}} \leq D
$$


for $\theta>1$ and some constant $D>0$ depending on $\theta$ and on a bound of the $L^{1}$ norm of $\mu_{1}^{n}, \mu_{2}^{n}$. Then the trace on $\partial \Omega$ of $u_{n}$ denoted $T u_{n}$ satisfies for every $q \in\left(1, \frac{N}{N-1}\right)$, the estimate

$$
\left\|T u_{n}\right\|_{W^{1-\frac{1}{q}, q}(\partial \Omega)} \leq \tilde{C}
$$

for some $\tilde{C}$ depending $C$ and $D$.

2.3. Passage to the limit in the regularized problem. We now come to the proof of Theorem 1.2 by passing to the limit $n \rightarrow+\infty$ in the weak formulation (2.13). From the previous sections, we have that up to subsequence

$$
u_{n} \rightarrow u \quad \text { weakly in } W^{1, q}(\Omega), q \in\left[1, \frac{N}{N-1}\right) .
$$

By continuity of the trace operator, we have

$$
T u_{n} \rightarrow T u \text { weakly in } W^{1-\frac{1}{q}, q}(\partial \Omega), q \in\left(1, \frac{N}{N-1}\right) .
$$

Then $T u_{n}$ converges strongly to $T u$ in $L^{q}(\partial \Omega)$ for some $q$ and then we have up to extraction of a subsequence

$$
T u_{n} \rightarrow T u \text { a.e. on } \Gamma_{2} \text {. }
$$

We have now to pass to the limit in the non linear term of the weak formulation (2.13). To do so, we adopt the strategy of [3] and have to prove equi-integrability of $\left|u_{n}\right|^{\gamma}$ on $\Gamma_{2}$. Let $t>0$ and $r>0$ and define

$$
\psi(s)=\left\{\begin{array}{c}
\inf \left((s-t)^{+}, 1\right) \text { if } s \geq 0, \\
-\psi(-s) \text { if } s<0,
\end{array}\right.
$$

Plugging $\varphi=\psi\left(u_{n}\right)$ in (2.13) , we are led to the following estimate

$$
\int_{E_{n, t+1}^{0}}\left|u_{n}\right|^{\gamma} \leq \int_{E_{n, t}^{0}}\left|\mu_{2}^{n}\right|+\int_{E_{n, t}}\left|\mu_{1}^{n}\right|+C \int_{E_{n, t}}\left|\nabla u_{n}\right|^{2} .
$$

where

$$
E_{n, t}=\left\{(x, t) \in \Omega \times \mathbb{R}^{+}|| u_{n}(x) \mid \geq t\right\}
$$

and

$$
E_{n, t}^{0}=\left\{(x, t) \in \partial \Omega \times \mathbb{R}^{+}|| T u_{n}(x) \mid \geq t\right\} .
$$

Using the $L^{q}$ bound for the gradient for some $q \in\left[1, \frac{N}{N-1}\right)$ gives that for some $\delta>0$

$$
\int_{E_{n, t+1}^{0}}\left|u_{n}\right|^{\gamma} \leq \int_{E_{n, t}^{0}}\left|\mu_{2}^{n}\right|+\int_{E_{n, t}}\left|\mu_{1}^{n}\right|+C \mathcal{L}\left(E_{n, t}\right)^{\delta}
$$


where $\mathcal{L}$ stands for the Lebesgue measure. Due to the $L^{q}$ bound of $\left|u_{n}\right|$, we have that

$$
\mathcal{L}\left(E_{n, t}\right) \rightarrow 0
$$

as $t \rightarrow+\infty$. Hence the quantity $\left|u_{n}\right|^{\gamma}$ is equi-integrable and Vitali's theorem ensures that

$$
\int_{\Gamma_{2}}\left|u_{n}\right|^{\gamma-1} u_{n} \rightarrow \int_{\Gamma_{2}}|u|^{\gamma-1} u
$$

This proves the desired result.

\section{The Degenerate CASE $\alpha \neq 0$ And PRoblem (1.2)}

We now come to the proof of Theorem (1.3). We will not give all the proofs since some of them are almost identical but emphasize the place where we loose regularity.

3.1. Regularization. Consider again smooth sequences $\left\{\mu_{1}^{n}\right\}_{n \geq 0},\left\{\mu_{2}^{n}\right\}_{n \geq 0}$ converging to $\mu_{1}$ and $\mu_{2}$ in the weak* topology in the sense described above. The regularized problem then writes weakly

$$
\int_{\Omega} d(x, \partial \Omega)^{\alpha} \nabla u_{n} \cdot \nabla \varphi+\int_{\Omega} \varphi \mu_{1}^{n}=\int_{\Gamma_{2}} \varphi \mu_{2}^{n}-\int_{\Gamma_{2}}\left|u_{n}\right|^{\gamma-1} u_{n} \varphi .
$$

Using the Lax-Milgram theorem in [7] together with a standard topological degree argument, one gets

Theorem 3.1. Let $\gamma>1$ and $\mu_{1}^{n}, \mu_{2}^{n}$ as before. There exists a weak solution $H^{1}\left(\Omega, d(x, \partial \Omega)^{\alpha}\right) \bigcap L^{\infty}(\Omega)$ of (3.21).

3.2. Estimates. The proof of the following lemma is identical to the proof of Lemma (2.2)

Lemma 3.2. Let $\gamma>1$ and $u_{n}$ be a weak solution of (3.21) with $\mu_{1}^{n}, \mu_{2}^{n} \in L^{2}(\Omega)$ and $L^{2}\left(\Gamma_{2}\right)$ respectively. Then for every $\theta>1$, there exists a constant $C>0$ depending on a bound of the $L^{1}$ norms of $\mu_{1}^{n}, \mu_{2}^{n}$ such that

$$
\left\|u_{n}\right\|_{L^{\gamma}\left(\Gamma_{2}\right)} \leq C
$$

and

$$
\int_{\Omega} d(x, \partial \Omega)^{\alpha} \frac{\left|\nabla u_{n}\right|^{2}}{\left(1+\left|u_{n}\right|\right)^{\theta}} \leq C .
$$

Before stating the lemma concerning the regularity on the gradient of $u_{n}$, we recall the Sobolev embedding proved in [7]. 
Theorem 3.3. (see [7]) Let $\Omega$ be an open set of $\mathbb{R}^{N}$ and $w$ an $A_{2}$ function; Then there exist constants $C>0$ and $\delta>0$ depending on $\Omega$ and the weight $w$ such that for all $u \in C_{0}^{\infty}(\Omega)$ and all $1 \leq k \leq \frac{N}{N-1}+\delta$ the following holds

$$
\|u\|_{L^{2 k}(\Omega, w)} \leq C\|\nabla u\|_{L^{2}(\Omega, w)}
$$

Remark that here the Sobolev exponent is not $\frac{2 N}{N-2}$ but $\frac{2 N}{N-1}+2 \delta$ and this is where we loose regularity in the estimates. The constant $\delta$ depends on the weight $d(x, \partial \Omega)^{\alpha}$ and the domain $\Omega$.

Lemma 3.4. Let $\mu_{1}^{n}$ and $\mu_{2}^{n}$ as before. Consider $u_{n}$ a weak solution of (3.21) such that

$$
\int_{\Gamma_{2}}\left|u_{n}\right|^{\gamma} \leq C
$$

for some constant $C>0$ depending on $\theta$ and

$$
\int_{\Omega} d(x, \partial \Omega)^{\alpha} \frac{\left|\nabla u_{n}\right|^{2}}{\left(1+\left|u_{n}\right|\right)^{\theta}} \leq D
$$

for $\theta>1$ and some constant $D>0$ depending on $\theta$ and on a bound of the $L^{1}$ norms of $\mu_{1}^{n}, \mu_{2}^{n}$. Therefore, we have that $u_{n}$ is bounded (in terms of $q, C, D)$ in $W^{1, q}\left(\Omega, d(x, \partial \Omega)^{\alpha}\right)$ for every $1 \leq q<\frac{N}{N-1}+\delta$.

Proof. We write, by Hölder inequality for $q \in[1,2)$

$$
\begin{gathered}
\int_{\Omega} d(x, \partial \Omega)^{\alpha}\left|\nabla u_{n}\right|^{q}= \\
\int_{\Omega} d(x, \partial \Omega)^{q \alpha / 2} \frac{\left|\nabla u_{n}\right|^{q}}{\left(1+\left|u_{n}\right|\right)^{q \theta / 2}} d(x, \partial \Omega)^{\alpha-q \alpha / 2}\left(1+\left|u_{n}\right|\right)^{\theta q / 2} \leq \\
\left\{\int_{\Omega} d(x, \partial \Omega)^{\alpha} \frac{\left|\nabla u_{n}\right|^{2}}{\left(1+\left|u_{n}\right|\right)^{\theta}}\right\}^{q / 2}\left\{\int_{\Omega} d(x, \partial \Omega)^{\alpha}\left(1+\left|u_{n}\right|\right)^{\frac{\theta q}{2-q}}\right\}^{\frac{2-q}{2}} \leq \\
D_{1}\left\{\int_{\Omega} d(x, \partial \Omega)^{\alpha}\left(1+\left|u_{n}\right|\right)^{\frac{\theta q}{2-q}}\right\}^{\frac{2-q}{2}} .
\end{gathered}
$$

We now use the Sobolev embedding (3.24) to estimate the last term. Since $u_{n}=0$ on $\Gamma_{1} \subset \partial \Omega$, we have the Sobolev embedding

$$
\left\|u_{n}\right\|_{L^{2 k}\left(\Omega, d(x, \partial \Omega)^{\alpha}\right)} \leq C\left\|\nabla u_{n}\right\|_{L^{2}\left(\Omega, d(x, \partial \Omega)^{\alpha}\right)}
$$

for all $1 \leq k \leq \frac{N}{N-1}+\delta$ for some $\delta>0$.

On the other hand from the inequality (3.25), we have that $\left\{\phi_{\theta / 2}\left(u_{n}\right)\right\}_{n}$ is bounded in $H^{1}\left(\Omega, d(x, \partial \Omega)^{\alpha}\right)$. By the previous embedding, the sequence $\left\{\phi_{\theta / 2}\left(u_{n}\right)\right\}_{n}$ is also bounded in $L^{2 k}\left(\Omega, d(x, \partial \Omega)^{\alpha}\right)$ and since, when $r$ is big enough, the function $\phi_{\theta / 2}(r)$ behaves like $r^{1-\theta / 2}$, we deduce that 


$$
\left|u_{n}\right|^{1-\theta / 2} \in L^{2 k}\left(\Omega, d(x, \partial \Omega)^{\alpha}\right)
$$

and then

$$
\left|u_{n}\right|^{2 k-k \theta} \in L^{1}\left(\Omega, d(x, \partial \Omega)^{\alpha}\right) .
$$

Therefore, the sequence $\left\{u_{n}\right\}_{n}$ is bounded in $L^{q}\left(\Omega, d(x, \partial \Omega)^{\alpha}\right)$ if

$$
\begin{aligned}
& \frac{\theta q}{2-q}=2 k-k \theta, i . e . \\
& q=\frac{2(2-\theta) k}{\theta+k(2-\theta)}>1
\end{aligned}
$$

for $1<\theta<2$. Taking $\theta$ arbitrarily close to 1 , one gets

$$
q<\frac{2 N+2 \delta(N-1)}{2 N-1+\delta}
$$

Since $d(x, \partial \Omega)^{\alpha}$ is integrable, one gets a bound for the last term in the previous range of $q$.

The previous theorem admits the following corollary by just using the trace embedding in [11].

Corollary 3.5. Let $\mu^{1} \mu_{2}^{n}$ as before. Consider $u_{n}$ a weak solution of (3.21) such that

$$
\int_{\Gamma_{2}}\left|u_{n}\right|^{\gamma} \leq C
$$

for some constant $C>0$ and

$$
\int_{\Omega} d(x, \partial \Omega)^{\alpha} \frac{\left|\nabla u_{n}\right|^{2}}{\left(1+\left|u_{n}\right|\right)^{\theta}} \leq D
$$

for $\theta>1$ and some constant $D>0$ depending on $\theta$. Then the trace on $\partial \Omega$ of $u_{n}$ denoted $T u_{n}$ is bounded in $W^{1-\frac{1+\alpha}{q}, q}(\partial \Omega)$ for every $1<q<$ $\frac{2 N+2 \delta(N-1)}{2 N-1+\delta}$.

The passage to the limit works exactly as in the previous section and this proves Theorem 1.3 .

\section{Extensions of THE PREvious RESUlT}

Thanks to the approach developped in [2], it would be possible to extend our results to more general problems, such as

$$
\left\{\begin{array}{c}
-\operatorname{div} A(x, \nabla u)=\mu_{1}, \text { in } \Omega, \\
u=0, \text { on } \Gamma_{1} \subset \partial \Omega, \\
A(x, \nabla u) \cdot \nu=\mu_{2}+h(u), \text { on } \Gamma_{2} \subset \partial \Omega
\end{array}\right.
$$


under structural assumptions on $A(x, \nabla u)$ (as to be monotone on the gradient term (like for instance the case of the $p$-laplacian) )and structural assumptions on $h$.

We refer the interested reader to [2] for the structural assumptions on the data. Remark that we can assume, thanks to the present work, degeneracy of the operator $A(x,$.$) close to the boundary.$

\section{REFERENCES}

[1] P. Benilan, H. Brezis, and M. G. Crandall. A semilinear equation in $L^{1}\left(R^{N}\right)$. Ann. Scuola Norm. Sup. Pisa Cl. Sci. (4), 2(4):523-555, 1975.

[2] L. Boccardo and T. Gallouët. Nonlinear elliptic and parabolic equations involving measure data. J. Funct. Anal., 87(1):149-169, 1989.

[3] L. Boccardo, T. Gallouët, and J. L. Vázquez. Nonlinear elliptic equations in $\mathbf{R}^{N}$ without growth restrictions on the data. J. Differential Equations, 105(2):334-363, 1993.

[4] H. Brézis and W. A. Strauss. Semi-linear second-order elliptic equations in $L^{1}$. J. Math. Soc. Japan, 25:565-590, 1973.

[5] L. Caffarelli and L. Silvestre. An extension problem related to the fractional Laplacian. Commun. in PDE, 32(8):1245, 2007.

[6] V. Chiadò Piat and F. Serra Cassano. Relaxation of degenerate variational integrals. Nonlinear Anal., 22(4):409-424, 1994.

[7] E. B. Fabes, C. E. Kenig, and R. P. Serapioni. The local regularity of solutions of degenerate elliptic equations. Comm. Partial Differential Equations, 7(1):77-116, 1982.

[8] T. Gallouët and J.-M. Morel. The equation $-\Delta u+|u|^{\alpha-1} u=f$, for $0 \leq \alpha \leq 1$. Nonlinear Anal., 11(8):893-912, 1987.

[9] N. S. Landkof. Foundations of modern potential theory. Springer-Verlag, New York, 1972. Translated from the Russian by A. P. Doohovskoy, Die Grundlehren der mathematischen Wissenschaften, Band 180.

[10] B. Muckenhoupt. Weighted norm inequalities for the Hardy maximal function. Trans. Amer. Math. Soc., 165:207-226, 1972.

[11] A. Nekvinda. Characterization of traces of the weighted Sobolev space $W^{1, p}\left(\Omega, d_{M}^{\epsilon}\right)$ on M. Czechoslovak Math. J., 43(118)(4):695-711, 1993. 